\title{
Un mártir extraordinario. El caso de Nagisa Kaworu en Shin Seiki Evangelion
}

\section{An Extraordinary Martyr. The Case of Nagisa Karworu in Shin Seiki Evangelion}

\author{
Roberto Jesús Sayar \\ Universidad de Buenos Aires \\ Universidad de Morón \\ Universidad Nacional de La Plata
}

Roberto J. Sayar (Haedo, Argentina, 1985) es licenciado y profesor secundario y superior en Letras de la Facultad de Filosofía y Letras - UBA; diplomado universitario en Asuntos Docentes por la Universidad de Morón y maestrando (en curso) en Literaturas Comparadas de la FaHCE - UNLP. Ha publicado varios capítulos de libros, una traducción y diez artículos sobre temas de su especialidad, que giran, por un lado, en torno al eje de la exégesis bíblica en la serie de textos de los Macabeos y por el otro en las relaciones que pueden rastrearse entre el mundo clásico y la producción cultural japonesa.

Fecha de recepción: 22 de abril de 2020

Fecha de aceptación definitiva: 6 de noviembre de 2020 


\title{
Resumen
}

A partir de las nociones del «ángel» como potencia intermediadora de la voluntad divina hacia los humanos y los tópicos de las primeras figuras de mártires que nos ofrece la literatura apócrifa (particularmente $I V$ Macabeos) será el propósito de este trabajo identificar en el personaje Nagisa Kaworu de la serie televisiva e historietística Shin Seiki Evangelion la adscripción a una y otra categorías como componentes fundamentales e indivisibles de su naturaleza, pues estas serían las que permitirían la adecuada comprensión de los discutidos momentos finales de dichas obras, que pecarían — según algunos - de una aparente e infundada oscuridad.

Palabras clave: Ángel, Evangelion, IV Macabeos, mártir, mass media.

\begin{abstract}
In light of the notions of the «angel» as an intermediary power of divine will towards humans and the topics of the first martyr figures that are brought to us by apocryphal literature (particularly by IV Maccabees), it will be this work's purpose to identify in Nagisa Kaworu, character of TV and comic series Shin Seiki Evangelion, its adscription to one and another categories as fundamental and undivided aspects of his nature, because such will allow the adequate understanding of the so debated final moments of these works, that - according to some people - would seem to be apparently and unjustifiably too dark.
\end{abstract}

Keywords: Angel, Evangelion, IV Maccabees, martyr, mass media.

\section{Cita bibliográfica}

Sayar, R.J. «Un mártir extraordinario. El caso de Nagisa Kaworu en Shin Seiki Evangelion», ", en CuCo, Cuadernos de cómic n.o 15 (2020), pp. 128-151. 
Valkyriae qua, sicut angeles, non semper vidi, sed amorem suum sentio

\section{Introducción}

¿Cómo aferrar lo inaferrable? ¿Cómo comprender lo incomprensible? Estas dos preguntas, cuyas respuestas distan sobremanera de rozar siquiera la sencillez, fueron las que seguramente se plantearon los pueblos antiguos a la hora de intentar comprender los fenómenos que se producían continuamente a su alrededor, sobre todo los naturales. Es decir, aquellos para los que, al menos de primera mano, no existía una explicación causal coherente, de no ser por la presencia de potencias superadoras de la capacidad humana que los controlaran. De esta forma, saber que estas tenían efectiva injerencia sobre hechos inalcanzables permitió además pensar que podían tener un papel en el devenir cotidiano puesto que, de no ser por su beneplácito, de una u otra forma nada existiría, dado que todo resulta inferior a dichas fuerzas, que ya a esta altura podremos calificar como divinas o al menos de naturaleza similar. Si, además, por otro lado, es lógico pensar que cada grupo humano posee y desarrolla su propia manera de ver el mundo que los rodea, en consecuencia, también será deducible que el ordenamiento, la forma y los alcances de estas fuerzas numinosas ${ }^{1}$ sean diferentes y penetren de maneras diversas la existencia, según el pueblo del que hablemos.

Ahora bien, si la pregunta se centrara en el modo en que podían explicarse o racionalizarse las calamidades que se sucedían cotidianamente, ya naturales, ya producto de su vida en sociedad con otros humanos, la situación puede tornarse insostenible. Efectivamente, las respuestas tentativas a esta pregunta exceden con mucho los límites de este trabajo. Por lo que nos limitaremos a decir que - circunscribiéndonos a una visión eurocéntrica - la paulatina identificación de Dios con la idea del Bien sumía a los pueblos en un atolladero metafísico y práctico, puesto que semejante asociación implicaría la imposibilidad de adosar aquellos sucesos de claro signo negativo a una entidad cuya ontología — de forma extremadamente simplificada- denota la asociación con una positividad extremadamente inalcanzable en su infinita perfección. La equivalencia entre estas dos ideas - la de la divinidad y la inmanencia de la suma de las virtudes en su sacra persona-implicó necesariamente que el concepto de «mal» y todos los elementos asociados a él habrían de penetrar en el mundo gracias a potencias del mismo tenor pero de distinto signo; esto es, divinidades indeterminadas que sin llegar a ser reconocidas como dioses, puesto que no son benéficas, tuvieran la posibilidad de ocupar el lugar de ellos y, en consecuencia, afectar de una manera similar la vida del hombre. De modo directamente proporcional, cuanto más fuertes

1 Отто, R. Lo santo. Lo racional y lo irracional en la idea de Dios. Madrid, Alianza, 1998, pp. 198-203. 
se consideraran estas potencias metafísicas, tendían a verse más alejadas de la cotidianeidad mundana. Por lo que un número insondable de seres intermedios fue creado para llenar ese espacio entre el Alto Cielo (o el Averno) y la secularidad terrena. De este modo, el transmitir o ejecutar las órdenes de las entidades divinizadas devino la función primigenia de las diferentes clases de dáimones, pensados para suplir este vacío categorial de forma tal que el mundo se fue llenando de ellos. El hombre, necesitado de esta intermediación, fue brindándoles con el tiempo tantas especificidades que culminarían formando una verdadera burocracia celeste. ${ }^{2}$

\section{¿Es posible pensar en una «angelología» oriental? Occidente, los mass media y el choque cultural}

\section{Metafísicas orientales}

En principio, resulta preciso aclarar que un ordenamiento semejante no solo se aplicará al ámbito mediterráneo, sino que serán todas las religiones — con mayor o menor intensidad, cualquiera fuera su grado de complejidad u organización — quienes harán aparecer en sus panteones figuras mediadoras entre los dioses y los hombres con la finalidad de que los designios de los primeros sean cumplidos por los segundos o bien que los ruegos del mundo terreno lleguen a oídos del mundo divino.

Dentro de las que parecerían no admitirlo en atención a su abierto y claro politeísmo, el shinto no es la excepción. Pues, a pesar de su marcado carácter animista, posee una clara jerarquía en la que los dioses del cielo, en un tiempo primigenio, hicieron entrega de ciertos lugares a deidades de la tierra quienes, a su vez, debían obediencia y respeto a sus equivalentes celestiales. ${ }^{3}$ La situación subsiguiente, según la que podría plantearse una verdadera topología divina, implicará sobre todo que cada región, elemento o situación de la vida diaria pueda vincularse con el representante de Amaterasu - la diosa solar, cabeza de todo el panteón - pasando necesariamente por todos los estados intermedios en pos de lograr siquiera acercarse a semejante divinidad. La persona imperial, receptáculo de la tradición divina en tanto parte del tronco familiar sagrado, en este contexto, se verá reducida a su faceta «mágica» ${ }^{4}$ puesto que el ritual de

${ }_{2}$ Cfr. las afirmaciones de Pérez Jiménez, A. y Cruz Andreotti, G. (eds.). Seres intermedios. Ángeles, Demonios y Genios en el Mundo Mediterráneo. Madrid, Ediciones Clásicas, 2000, p. 3.

3 Breen, J. y Teeuwen, M. A New History of Shinto. Chichester, Wiley-Blackwell, 2010, p. 27. Lo mismo sostienen los editores castellanos de Kojiki, libro que narra los orígenes y el desarrollo del Japón como unidad étnica, política y religiosa. Rubio y Moratalla afirman que esto se hizo para imbricar a todos los clanes dominantes de la política nipona dentro de una historia sagrada que legitimara el naciente poder político del joven estado japonés. En Rubio, C. y Moratalla, R. T., «Introducción», en Oo no Yasumaro (comp.). Kojiki, Madrid, Trotta, 2008. p. 18-19.

${ }^{4}$ Ferretti, V. «Emperor Reigen and the Change in Court-Bakufu Relations since the Tenna to the Kyōtō Era», en East and West, n. ${ }^{\circ} 1 / 2$ (1996), pp. 167-68. 
la corte se convertirá gradualmente en el modelo de adoración para todas y cada una de las Ocho Millones de Deidades. ${ }^{5}$ Ambos, reuniendo en sus esfuerzos las corrientes binarias del Tao, tan valoradas por la literatura china y las lógicas confucianas que constituyeron los textos fundantes, conformarán una simbología lo suficientemente fuerte que será considerada inamovible. La maniobra, de este modo, implicará colocar en una primacía indiscutida a la familia del Sol y relegar a los dioses relacionados con ella (que podrían ser potencialmente peligrosos) a una situación claramente desventajosa. No solamente mediante derrotas en combates abiertos plagados de simbolismos chamánico-mágicos, ${ }^{6}$ sino también a través de elementos desacralizados que declaran intrínsecamente su condición subordinada. Así, su importancia será gravemente disminuida en pos de la carga fuertemente negativa que poseen ambos elementos y se podrá aprovechar totalmente la lógica parental típica del orden social chino, ${ }^{7}$ tanto por la familia gubernamental como por todos aquellos clanes que buscan arrebatarle de manera legítima ese sitio preeminente.

Además, el hecho de que este conjunto de creencias se caracterice no solo por poseer una serie de divinidades que se manifiestan con preferencia en elementos de la naturaleza, sino también por no implicar necesariamente ningún código ético-moral al cual adscribirse, ciertamente ayuda a que el fenómeno religioso en el Japón goce de una penetración particular. Ahora bien, la vinculación de la sociedad con una corriente de pensamiento sacro determinado no acaba en este punto, ya que es posible entender los entrecruzamientos que se dan entre el culto tradicional y aquellas creencias que le son más usuales (como el budismo, el propio confucianismo o el taoísmo), de manera similar a la existencia de los miles de dioses del shinto con respecto a la deidad solar. Es decir: que las diferentes manifestaciones metafísico-religiosas vendrán a completar los intersticios que el «camino de los dioses» haya dejado - intencional o involuntariamente- en el entramado de creencias de la nación. Es en este particular contexto que el cristianismo llega a convertirse en otro camino diverso para pensar la intermedialidad entre el hombre y el Cielo.

\section{La inserción del cristianismo}

En efecto, desde la inserción de este credo de manos de misioneros jesuitas comandados por Francisco Javier (1549), la fe de Cristo ha sido una presencia constante e inconsciente en la sociedad nipona de modo que su simbología ha llegado a adquirir un estatus místico y sobre todo simbológico cuasi equivalente al que ostenta el culto de

\footnotetext{
5 Teeuwen, M. y Schied, B. «Tracing Shinto in the History of Kami Worship», en Japanese Journal of Religious Studies, Vol. 29/3-4 (2002), p. 205.

${ }^{6}$ Cfr. Naumann, N. Antiguos mitos japoneses. Barcelona, Herder, 1999, p.138-139.

7 Cfr. Levi, J. Los funcionarios divinos. Política, despotismo y mística en la China antigua. Madrid, Alianza, 1991, p. 20-21.
} 
los kami. El punto nodal de esta recepción se concentrará en la existencia de la deidad única y lo que implica esta singularidad en el ordenamiento y el devenir de la vida. El camino que escogió el cristianismo para resaltar este punto, poco después de la partida de los primeros evangelizadores, fue específicamente «adecuarse (accomodatio) a la cultura nativa y $[\ldots]$ sin destruir nada de ella, trasplantar en la cúspide de esa cultura la superior cultura cristiana». ${ }^{8}$ Así, la concepción de una vida ultraterrena diversa a la planteada por las corrientes budistas desplegadas en el archipiélago será un rasgo de particular importancia para lograr que la nueva fe pudiera insertarse exitosamente entre los numerosos caminos ya citados que ligaban al hombre con sus dioses. La variable celestial adquirirá, en consecuencia, una profundidad esencial dentro de un ecosistema religioso tan atravesado por la idea de la finitud y de la fugacidad de los instantes. ${ }^{9}$ Más allá de que las diferencias subsistan en el Japón moderno, y algunos grupos especialmente tradicionalistas continúen considerando al cristianismo como una religión amenazante, tanto para la cultura del archipiélago como para la adhesión patriótica a Japón como una nación intrínsecamente shintoísta — en tanto el emperador continúa disponiendo de los recursos del Estado para llevar adelante ceremonias que, en esencia, pertenecen a la vertiente más antigua del «camino de los dioses»—, ${ }^{10}$ la carga esotérica del cristianismo no hará otra cosa sino colocarse a sí misma en el sitio que las dos grandes religiones de las islas han dejado aparentemente insatisfecho. En consecuencia, la existencia de una serie de potencias supraterrenales que consigan mediar exitosamente entre el inaccesible más allá y la realidad cotidiana ejercerá un rol más abarcativo (en razón de su dilatado número) que muchas de las corrientes budistas que se desperdigaron entre los nipones.

\section{Intersecciones en los mass media}

En virtud de sus peculiares características compositivas, es posible comprender que es en el ámbito del manga (漫画) y el anime (アニメ), contraparte animada de las páginas de la historieta, en donde este hecho puede observarse con mayor énfasis. En vista de que este grupo íntimamente relacionado de mass media tendrá una particular penetración e interrelación con los gustos del gran público, su análisis podrá revelar de qué modo la vida religiosa del Japón moderno interpela a sus practicantes/consumidores. Arista analítica que se justifica, además, en el hecho de que estos productos culturales no solamente sabrán interpretar las problemáticas particulares de su públi-

\footnotetext{
${ }^{8}$ Miyazaki, K. «Roman Catholic Mission in Pre-Modern Japan», en Mullins, M. R. (ed.). Handbook of Christianity in Japan, Leiden-Boston, Brill, 2003, p. 8. El paréntesis del latín (y las redundancias terminológicas) son del original.

9 Heine, S. «From Rice Cultivation to Mind Contemplation: The Meaning of Impermanence in Japanese Religion», en History of Religions, n. ${ }^{\circ}$ 30/4 (1991), pp. 373-403.

$10 C f r$. Breen, J. «Shinto and Christianity: A History of Conflict and Compromise», en Mullins, M. R. (ed.). Handbook of Christianity in Japan, Leiden-Boston, Brill, 2003, pp. 268-270.
} 
co, sino abordarlas de manera que no sean percibidas como el motivo principal para legitimar su consumo. ${ }^{11}$ Es cierto que están sujetos a la ley de oferta y demanda y al mismo tiempo tienden a imponer símbolos de fácil universalidad, ${ }^{12}$ como modo de sensibilizar a sus consumidores en su enfrentamiento con el mundo. ${ }^{13}$ Para lo cual es acertado recordar que es en el manga y en su público donde parece que la afirmación de Eco que asegura que

el cómic es un producto cultural disfrutado y juzgado por un consumidor que [...] está especificando la propia solicitud [de disfrute] en esta dirección, pero que aporta a esta experiencia de fruición su experiencia entera de hombre educado asimismo para la fruición de otros niveles ${ }^{14}$

se lleva a la práctica de manera total y acabada. En Japón, un lugar donde más del $25 \%$ del mercado editorial está dedicado a ellos, ${ }^{15}$ los cómics no son solo un medio de entretenimiento sino, como siempre se ha defendido, una forma de arte de pleno derecho, compatible y coextensiva con todos los demás modos de expresión de la creatividad humana, ya inseparable - e incluso indistinguible - de cualquier otra forma artística. Y, en carácter de tal, será entre sus páginas (y sus consecuentes fotogramas) que la simbología cristiana ha sido explotada de manera singular. El punto culminante de este empleo será el uso ecléctico que de ella se hace en la serie animada y la historieta Shin Seiki Evangelion. La obra basa su trama en un interesante entrecruzamiento de referencias judeocristianas con rasgos típicos de la religiosidad, la ética y la moral nipona a niveles extremos.

Por lo que intentaremos demostrar en este trabajo que una de las figuras principales de la obra, que luego devendrá el antagonista más importante, corporiza en sí un caso evidente de martirio según los cánones que llegan hasta nosotros desde los presentados por la literatura sacra judeocristiana, tanto en sus ejemplos católicos ${ }^{16}$ como en los ortodoxos, ${ }^{17}$ en parte gracias a que su entidad humana no es otra cosa que la de un ángel presuntamente semítico que ha sido genéticamente modificado para encarnar en un cuerpo. En consecuencia, será posible comprender y sostener que, tal como sus

${ }^{11}$ Cfr. Ingulsrud, J. E. y Allen, K. Reading Japan Cool. Patterns of Manga Literacy and Discourse. New York, Lexington Books, 2009.

12 Eco, U. Apocalípticos e integrados. Buenos Aires, Sudamericana, 2013 [1964], pp. 65-66.

13 Ibid., p. 73.

14 Ibid., p. 85.

15 Совоs, T. «Animación japonesa y globalización: la latinización y la subcultura otaku en América Latina», en Razón y palabra, n. 72 (2010), p. 5. Disponible en: http://www.razonypalabra.org. $\mathrm{mx} / \mathrm{N} / \mathrm{N} 72 /$ Varia $72 / 32$ Cobos 72.pdf

162 Mac. 6-7, en Rahlfs, A. (ed.). Septuaginta, id est Vetus Testamentum graecae iuxta LXX interpretes Vol. 1-2. Stuttgart, Württembergische Bibelanstalt, 1971.

174 Mac. 5-16, en Rahlfs, A. (ed.). Op. cit. 
antecesores librescos, devenga el portador de una ejemplaridad positiva destacable cuyos efectos tendrán una doble funcionalidad. Por un lado, serán estrictamente necesarios intradiegéticamente para la adecuada comprensión del enrevesado final y, por el otro, extradiegéticamente, para la instalación de una interpretación del cristianismo autóctono mucho más alejada de las posturas tradicionalmente sostenidas y, por ende, más cercana a la simplificación sincrética de la mayoría de las creencias que convivieron en el Japón de fines del siglo pasado.

\section{Angelología nipona y manipulación genética: Preludios del Tercer Impacto}

\section{Contextos e intertextos}

La historia que nos ocupará a lo largo del presente trabajo, Shin Seiki Evangelion (lit. «Evangelio del nuevo siglo»), es una serie de televisión animada que salió al aire por vez primera en las pantallas de TV Tokyo el miércoles 4 de octubre de 1995 para concluir su emisión, luego de los veintiséis episodios planeados, el 27 de marzo de 1996. En ella se presenta una historia fuertemente distópica donde la humanidad trata de reconstruirse después de un cataclismo mundial. Quince años después de este evento, lo poco que queda en pie de las islas de Japón se ve invadido por un ser biológico gigantesco al que las fuerzas de seguridad denominan «Ángel» ${ }^{18}$ y a quien, llamativamente, parecen haber estado esperando. El conflicto de la serie se plantea cuando el protagonista, Shinji Ikari, al encontrarse con su padre - a quien no ve desde hace diez años - se ve impelido a abordar un robot gigante (el Evangelion) y así poder salir a pelear en igualdad de condiciones contra este Ángel. Partir de estas premisas implicará por un lado la generación de una posición conflictiva entre los integrantes de la agencia NERV y quienes buscan oponérsele, en tanto estos últimos pueden identificarse como fuerzas que no deberían ser enfrentadas. Por otro lado, la confrontación con el Cielo desatará las aristas más oscuras de la psiquis de la raza humana, en tanto se devele que el meollo del argumento incluye forzar a la especie a acceder a un ulterior estado evolutivo mediante el cual cada uno de los individuos dejarán de existir como entes separados para pasar a constituir un único organismo. Como se puede deducir de estas escuetas líneas, el papel de los ángeles - y la manera de ser interpretados- en la economía de la serie no es menor y, como ha sucedido desde que esta viera la luz, se presta a varias lecturas que la obra misma no deja esca-

18 Seguimos, en este punto y en muchas de las menciones a palabras no-japonesas, el criterio del traductor al castellano rioplatense de la historieta (SADAmoto, Y. y Gainax. Evangelion. Buenos Aires, Ivrea, 2004. Traducción de Agustín Gómez Sanz), quien indica que «a partir de que en la propia serie original y en el resto de la gráfica relacionada [...] escriben en inglés "ANGEL" en letras romanas occidentales, todas las traducciones de la serie han tomado este término para nombrarlos» (Sadamoto, Y. y Gainax. Op. cit., 3.25). Al momento de citar la obra impresa lo haremos por el número de los capítulos aparecidos en el Shonen Ace (y no por tomo). Luego del número de capítulo, tras un punto, aparecerá el número de página. 
par, puesto que hasta el propio Shinji, acuciado por las dudas, llega a expresar que «los ángeles son buenos, ¿por qué tenemos que llamar "ángel" a nuestro enemigo, por qué tenemos que luchar contra él?». ${ }^{19}$

Del mismo modo, en la historieta que procede de la versión animada, el papel de los ángeles fluctúa en una doble lectura según la cual no solo están resaltados como enemigos, sino que, además, su cualidad divina está destacada por varios elementos que aparecen en la trama de un modo particularmente subrepticio. Es decir, o se los nombra como si no tuvieran mayor importancia, o se los pretende accesibles tan solo a aquellos lectores que poseen una cultura judeocristiana avanzada. Es cierto que el lector modelo ${ }^{20}$ que plantea Evangelion no lleva implicado necesariamente ser católico (o judío) practicante ni mucho menos un erudito en angelología o en los dilemas de la existencia humana (psicológica y filosóficamente hablando). De todos modos, no es posible soslayar que difícilmente se alcanzaría el nivel de comprensión aparentemente pretendido por sus autores si no suponemos en primera instancia que el lector (y mucho más el televidente) de hace casi treinta años debía al menos tener una mínima noción de la naturaleza angélica y su vinculación con lo divino. Es en este punto, precisamente, donde la índole sincrética y - mínimamente- ecléctica de la concepción nipona de las religiones organizadas, sobre todo de las que han marcado el devenir metafísico en su territorio, adquirirán una importancia tan superlativa como necesaria para la adecuada comprensión de las diversas partes componentes de la serie y, sobre todo, aquellas relativas a la concepción cristiana del universo.

\section{Ángeles y demonios}

Inicialmente, ese conocimiento es necesario para poder decodificar a los robots — que alcanzarán una primacía equivalente a la de quienes los pilotan- como los demonios tradicionales de las creencias niponas $(O n i \text { 鬼 })^{21} \mathrm{y}$, consecuentemente, entender así por qué se busca que suceda lo que finalmente sucederá, esto es, el Plan de Consumación de la Humanidad: el siguiente estado evolutivo al que referimos anteriormente. Los ángeles, en consecuencia, no serán solo potencias divinas capaces de un nivel destructivo alarmante sino, sobre todo, seres plausibles de tomar decisiones, errar y llevar a cabo actividades específicas, dependientes o no de sus vínculos con los dioses. Caracterización singular que se desarrollará mucho más enfáticamente en la versión impresa de esta historia, a pesar de las acotaciones semántico-expresivas que implica

${ }_{19}$ Ep.11.11:27. Las veces que hagamos referencias a la serie animada de Evangelion (en adelante Eva) lo haremos indicando en primer lugar el número de episodio (Ep.) y, seguido de un punto, el momento de ese capítulo en el que la cita tiene lugar. Shin Seiki Evangelion y todas sus marcas derivadas son (C) GAINAX/Khara y se referencian aquí a mero título científico/divulgativo.

${ }^{20}$ Eco, U. Op. cit., pp. 69 y ss.

2186.12 en Sadamoto, Y. y Gainax. Op. cit. 
la opción por el papel y la explosión de recursos visuales típicos de esta clase de productos gráficos. Confines que, por otro lado, habilitarán un desarrollo más profundo de la mayoría de las figuras intervinientes en la diégesis en tanto es posible que dispongan de un espacio más dilatado que la extensión de un simple capítulo televisivo. Cierto es que en el contexto del celuloide es posible ahondar en todos los elementos paratextuales que profundizan el decir y el accionar asociado a cada uno de ellos, como la música, los efectos de luz o los tonos de voz que les serán específicos. Pero, al erigirse como el contrapeso necesario a la reducción temporal que sufren algunos eventos particularmente centrales en la evolución de la trama, esa carga semántica será únicamente un elemento cuya importancia deberá ser entendida en razón a la diversa manera en que se escenifican los silencios y las palabras en el material fílmico. La historieta, en un contexto analítico puramente textual, será entonces el medio idóneo para realizar una comparación de tipo genético, que nos permitirá abordar en conjunto tres construcciones tipológicas que, a pesar de su enorme distancia temporo-espacial, comparten más de una arista de contacto: la de los ángeles de la tradición medioriental, los mártires de impronta judeohelenística y, puntualmente, el último enemigo de la lista que Gendo Ikari y SEELE guardan bajo el máximo secreto: Nagisa Kaworu (渚力习 ル), el duodécimo ángel.

Gráficamente hablando, no aparenta cualidades extraordinarias más allá de su cabello gris y ojos excesivamente claros, características sustanciales que le hará decir a Shinji - tras su primer encuentro- que lo que siente al verlo «ya lo sintió antes, la primera vez que vio a Ayanami». ${ }^{22}$ Acto seguido, se presenta en el cuartel general de NERV (la agencia que desarrolló los Evangelion) y todas las personas que lo van encontrando en su camino sienten, tan solo al verlo, pensamientos o comportamientos extraños relacionados con su presencia en un ambiente que nunca terminan de entender como suyo, tanto como lo es de los primeros tres Children. ${ }^{23}$ Incluso Shinji alberga emociones no del todo amables hacia su persona, dado que el Quinto elegido parece desconocer toda sensibilidad humana: no demuestra el mínimo aprecio por ninguna forma de vida ${ }^{24} \mathrm{y}$ desconoce los límites de la intimidad ${ }^{25} \mathrm{y}$ el respeto por los espacios personales; ${ }^{26}$ cosas estas carísimas a la mentalidad nipona. Por último, y para reseñar la singularidad de este personaje, Rei Ayanami, también piloto de Evangelion, destaca que, a pesar de parecerse mucho, no son iguales. ${ }^{27}$ Con lo que, además de rebatir el sentimiento de Shinji sin que este lo sepa, marca una distancia insalvable entre ambos que ella demostrará en un acto de inmolación supremo al sacrificar su vida para

\footnotetext{
22 57.19, en idem.

${ }^{23}$ Cfr. 58.15; 58.20-21, en idem.

24 57.16, en idem.

25 58.14, en idem.

${ }^{26} 62.18$, en $i d e m$.

27 62.12, en idem.
} 
detener el avance de un ángel. ${ }^{28}$ Esto hará que Shinji albergue un gran resentimiento hacia Kaworu, ${ }^{29}$ cuyo momento álgido llegará instantes antes de que este último revele su verdadera naturaleza. ${ }^{30}$

\section{Kaworu como ángel: Zoroastro, Henoc y la voluntad del sacrificio}

\section{Angelologia hebrea y sus influencias}

Tal y como se ha adelantado a lo largo de los párrafos precedentes, Nagisa es también - además de piloto de Evangelion en tanto se lo considera un ser humano apto para tal menester- el último ángel de los que a lo largo de toda la serie han atacado Tokyo-3. $\mathrm{Su}$ identidad en cuanto tal es la de Tabris; nombre que en el manga se utiliza tan solo una vez como manera de recalcar la misión que tiene asignada, esto es, iniciar el Tercer Impacto (evento que tendría como consecuencia el comienzo del Plan de Consumación de la Humanidad). Este ángel, se dice, proviene de la tradición judeo-cristiana y presidiría la sexta hora de cada día, el libre albedrío y la autodeterminación. Analizaremos estas esferas de acción un poco más adelante e intentaremos ahora dilucidar su trasfondo mítico-religioso. Las fuentes a las que hemos tenido acceso no listan ningún texto de procedencia hebrea que haga referencia a tal criatura, ${ }^{31}$ pero sí hemos podido notar que un «ser intermedio» ${ }^{32}$ de este nombre aparece entre las entidades pertenecientes a la religión asirio-babilónica ${ }^{33}$ y luego pasa a formar parte del panteón zoroástrico con los mismos atributos. ¿Es posible, entonces, que quien pertenece a una corriente religiosa determinada se haga parte de otra serie de lógicas cultuales que pueden, o no, tener una relación directa entre sí? Para dilucidarlo es preciso recordar que, así como

el zoroastrismo y el judaísmo tenían varios puntos en común (como por ejemplo la creencia en ángeles), es probable que la religión persa influyera en la evolución del judaísmo. Después de todo, los persas habían devuelto a los judíos a Jerusalén e Israel siguió siendo un Estado cliente durante mucho tiempo. ${ }^{34}$

${ }^{28} 65.22$ y ss., en idem.

2966.15 , en idem.

30 71.13-16, en idem.

31 Ver, entre otros, Davidson, G. A Dictionary of Angels, including the fallen angels. New York, The Free Press, 1966, p. 83; Kelly, S. y Rogers, R. Who in hell. A guide to the whole damned bunch. Michigan, Villard, 1996, p. 219 y BANE, T. Encyclopedia of Demons in World religions and cultures. Jefferson, McFarland, 2012, p. 308. Todos ellos recopilan el dato de un texto de Apolonio de Tiana cuya versión nos es inaccesible. La que lista el TLG (Boll, F. [ed.] Codices germanici [Catalogus codicum astrologum Graecorum 7], Brussels, Lamertin) no lo consigna entre los ángeles de las horas.

32 Pérez Jiménez, A. y Cruz Andreotti, G. Op. cit.

33 Cfr. Vitaliano, D. Legends of the Earth. Bloomington, Indiana University Press, 1973, pp.161-162.

${ }^{34}$ Mann, M. Las fuentes del poder social, I. Madrid, Alianza, 1991, p. 349. 
Por lo tanto, resulta fuertemente plausible que el movimiento sociohistórico previo, que aconteció de forma inmediatamente posterior a la conquista persa del Imperio Asirio, haya podido retomar ciertos elementos sagrados desde los ritos y las costumbres de sus ahora subyugados. Para comprender semejante absorción simbólica, se debe tener en cuenta que

Los desterrados de la época asiria eran mucho más numerosos, y fueron dispersados por varias provincias, introduciendo en su lugar desterrados de las otras provincias, lo que produjo una simbiosis a escala social o familiar (más o menos forzosa) y un consiguiente sincretismo religioso. ${ }^{35}$

Por lo que, teniendo en cuenta ambas cosas, no parece muy descabellado que, consolidado el asentamiento del pueblo de Israel en Canaán, un djinn zoroástrico haya retomado su funcionalidad y su ubicación en la economía sagrada y aparezca ahora como un ángel hebreo o - más probablemente- como una hipóstasis ${ }^{36}$ del Altísimo que solo en la época helenística y durante el período del Segundo Templo haya «recuperado» su estatus angélico. Además, en relación con esto, incluso unas criaturas de esta misma naturaleza como los querubines tienen relación con un cúmulo de creencias ajeno al hebraísmo, puesto que derivan directa o indirectamente de los toros alados que resguardaban los templos de Asiria y luego de Persia. ${ }^{37}$ Además, como estos kerubu (término sumerio que designa a dichos seres) también poseen una función similar a la de los mensajeros celestiales hebreos, es decir, la intercesión por el ser humano ante la divinidad y su protección, es posible entender más claramente las palabras de Dios cuando amonesta a los ángeles que han pecado. Es decir, estas serían un modo patente de reconocer el hecho de que, como estos ángeles fueron primitivamente dioses pertenecientes a otras creencias, no solo fueron transformados en aspectos esenciales de Yahvés ${ }^{38}$ si no que se tendió a hacerlos parte del «séquito divino» del Altísimo y a encargarlos del culto celestial. ${ }^{39}$ Así lo afirma, entre muchas otras fuentes, el apócrifo Libro de Henoc, cuando, en medio del episodio del nacimiento de los gigantes, el Omnipotente les dice a sus servidores que «a vosotros corresponde interceder por los humanos y no a los humanos por vosotros». ${ }^{40}$

35 Liverani, M. El antiguo oriente. Barcelona, Crítica, 1995, p. 535.

${ }^{36}$ López Salvá, M. «Demonios y espíritus en las religiones orientrales primitivas», en PÉrez Jiménez, A. y Cruz Andreotti, G. (eds.). Seres intermedios. Angeles, Demonios y Genios en el Mundo Mediterráneo. Madrid, Ediciones Clásicas, 2000, p. 42.

37 Giovino, M. The Assyrian Sacred Tree: A history of Interpretations. Fribourg/Göttingen, Academc Press Fribourg/Vandenhoeck \& Ruprecht, 2007, p. 41.

${ }^{38}$ López Salvá, M. Ibid.

39 Leibovici, M. «Génies et démons en Babylonie» en VV.AA. Génies, anges et démons. Paris, Seuil, 1971.p. 124.

${ }^{40}$ Hen.15.2 en VV.AA. Apócrifos del Antiguo Testamento IV: Ciclo de Henoc. Madrid, Cristiandad, 1984. 
En consecuencia, esta parece ser una de las asimilaciones posibles de las divinidades ajenas, siendo la otra, como puede inferirse, la de demonizarlos y alejarlos por siempre de la posibilidad de redimirse ante su Creador. Cabe recordar que el propio Talmud reconoce que «los nombres de los ángeles fueron traídos por los que retornaron de Babilonia», ${ }^{41}$ por lo que es preciso tener siempre presente que las criaturas que rodeaban a Yahvé podían sentirse empequeñecidos con razón frente a Él —en tanto que ingresan al conjunto de las entidades sacras mucho más tarde que el resto de las cohortes celestiales y, por lo tanto, les es imposible intentar alcanzar su alto sitial—. Inseguridad que, finalmente, podrá justificar el por qué a estos ángeles se los encuadró en la categoría de «sirvientes» negándoles, casi, toda posibilidad de autodeterminación por fuera de su condición efectiva de servicio: su procedencia y larga tradición en religiones claramente politeístas no los hacía totalmente aptos para someterse por propia voluntad a los arbitrios del Todopoderoso.

\section{Consecuencias del libre albedrio}

Por eso, que el ángel que cierra el arco del enfrentamiento con el Cielo patrocine particularmente el ámbito de las elecciones y las opciones libres no resulta un dato menor. Una afirmación semejante parecería improcedente puesto que los ángeles —como se afirmó anteriormente- se caracterizan ante todo por el servicio y la mediación de Dios ante el ser humano. ${ }^{42}$ Pero, de desconocerse tal filiación y ajustarse únicamente a lo que el personaje demuestra en escena, no es imposible dilucidarlo, puesto que no es únicamente su propio nombre lo que destacaría su costado obediente en contraste a su arista humana, que tradicionalmente sería más propensa a la rebelión. En múltiples ocasiones se hará mención a la sumisión a sus superiores que lo caracterizará hasta el momento final de la batalla: tanto por someterse al escaneo de su personalidad para la construcción del Dummy Plug de los Evangelions construidos en masa ${ }^{43}$ como por no develar su propia naturaleza angélica en su enfrentamiento con Armisael ${ }^{44} \mathrm{u}$ obtener el control del Segundo Evangelion para cumplir con los propósitos de SEELE. ${ }^{45}$ Sin embargo, en el momento de su revelación saldrán a la luz sus verdaderas capacidades y posibles intenciones, que propiciarán su postrera entrega. Resulta útil retomar en este momento el episodio de los gigantes - citado anteriormente-, que ya en el primer libro de la Biblia presenta una caída de los ángeles puesto que algunos de ellos «miraron con deseo a las hijas de los hombres». ${ }^{46}$ Dicho evento será retomado y am-

${ }^{41}$ Leibovici. Op. cit., p. 133.

42 Cfr. Trigger, B. Understanding Early Civilizations. A Comparative Study. Cambridge, Cambridge University Press, 2003, pp. 270 y ss.

43 48.20-22 en Sadamoto, Y. y Gainax. Op. cit.

4464.13 en idem.

4572.8 en idem.

${ }^{46}$ Gen. 6.1-4, en Rahlfs, A. (ed.). Op. cit. 
pliado por el redactor del Libro de Henoc, quien no solo explica que los seres celestiales tienen deseos personales sino que, incluso, estas apetencias pueden desviarse de la voluntad de Dios. En consecuencia, los infractores pueden ser castigados ${ }^{47}$ e incluso encarcelados puesto que, por lo general, estos deseos traen aparejada una revelación mayor de la sabiduría celestial, ${ }^{48}$ depositada de forma estrictamente limitada en los mismos ángeles. $\mathrm{Al}$ comportarse entonces «como si fueran el Señor» ${ }^{49}$ se harían merecedores de una consumación ${ }^{50}$ para quitar de la Tierra todos los frutos derivados de tales apetencias y funestos para los planes divinos. La libertad de elección, entonces, no es algo de lo que los ángeles carezcan sino que sería un rasgo común a todos ellos debido a que distinguiría a los ángeles buenos de los caídos. Los primeros serán los que eligen permanecer fieles a los mandatos recibidos, y los segundos los que dejaron que sus propias decisiones fueran más importantes que la voluntad divina. En nuestro caso particular, y dado que la propia serie ya cuenta con un ejemplo de ángel caído como parte de los enemigos, no parece inadecuado postular que el último de ellos sea el que tenga más desarrollada tal capacidad de autodeterminación.

\section{De la obediencia angélica a la entrega del martirio: Eleazar como modelo de comportamiento humano}

En efecto, este libre albedrío será el que le dará a nuestro ángel la primera característica que lo colocará como un presunto mártir. Quien está dispuesto a ofrendar su vida por las ideas que defiende debe ser, por un lado, plenamente consciente de esas nociones y, por el otro, tener en mente que es preferible morir y no cejar en la propia convicción que vivir a costa de su pérdida. En nuestro caso, Kaworu conoce con seguridad tanto lo que sucederá en caso de que obedezca el mandato por el cual debía infiltrarse en el nivel más recóndito de la base de NERV — evento que supuestamente desencadenaría el Tercer Impacto- como lo que pasaría de no acatar dicha orden. En efecto, lo expresará de la siguiente manera:

Puedo elegir desafiar a mi destino [...]. Si hago eso, los viejos no me lo van a dejar pasar. Ellos seguramente van a eliminarme [...]. Entonces... si causo el Tercer Impacto o no... de cualquier forma voy a desaparecer como individuo. La única libertad real que tengo es elegir la forma en la que voy a morir. ${ }^{51}$

${ }^{47}$ Hen. 13.5, en VV.AA. Op. cit.

${ }^{48}$ Hen.21.10, (en idem); cfr. 2Pe. 2.4 (en Rahlfs, A. (ed.). Op. cit.).

${ }^{49}$ Hen. 68.5, en VV.AA. Op. cit.

${ }^{50}$ Hen. 10.2, en idem. Es llamativa la utilización de este término por el redactor del Libro de Henoc (en

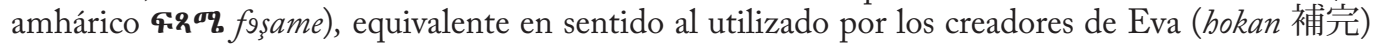
para describir el futuro de la humanidad. Quizás esto ayude a entender el plan como una necesidad de «recreación del mundo» análoga a la sucedida en los tiempos de Noé.

51 73.23-24, en Sadamoto, Y. y Gainax. Op. cit. 
Esto no tendría mayor significación más allá de la de un suicidio, si no fuera porque será posible comprender la cabal significación de una declaración semejante si se la intersecta con las últimas palabras que emite su contraparte animada. Entonces, mientras la mano del Evangelion.01 lo mantiene sujeto, le dirá a Shinji con una sonrisa en los labios ${ }^{52}$ que:

Mi destino es vivir eternamente aunque los seres humanos se sigan destruyendo eternamente entre sí. [...]. Elimíname, si no lo haces los eliminados serán ustedes [...]. Mi vida será el precio que pagaremos para evitar la destrucción de todo el mundo.

Por lo tanto, remarcar la notoriedad que implica que esta existencia carnal sea un precio a pagar para evitar el cataclismo planetario y que, efectivamente, elija hacerlo - ya sea en consideración a Shinji o como una venganza personal en contra de SEELE - no será para nada inocente. Un concepto expiatorio similar puede retrotraerse hasta las visiones deuteronómicas de la historia, plasmadas en algunos textos veterotestamentarios, en donde los pecados a expiar son los de todo el pueblo judío. Las lógicas textuales de esta corriente narrativa explicitan la cadena de hechos que desata la transgresión de la ley y resaltan, al mismo tiempo, la necesidad de una figura sacrificial lo suficientemente simbólica en cuanto a sus características ético-morales para lograr sobreponerse al alejamiento divino y sus consecuencias. De esta manera, el pecado propicia no solo que Dios se aleje sino que incluso dé vía libre a los enemigos de Israel para que lo ataquen exitosamente y lo sometan a innumerables vejaciones. ${ }^{53}$ Así, la figura del mártir se alzaría para, a través de su conducta amoldada de manera perfecta a las normativas recibidas, mover a la divinidad a compasión y que, entonces, ceda en su furor contra la colectividad.

En palabras de Eleazar, el anciano protagonista del Libro IV de los Macabeos, se pide que Dios «ha[ga] de su sangre su purificación y to[me] su vida en lugar de la de ellos», ${ }^{54}$ siendo «ellos» todos los miembros del grupo que escogieron alejarse de las normativas ancestrales. De otro modo, el mártir no existiría. Es decir, tanto si la muerte se produjera por algún motivo que no sea la preferencia por ella en lugar de una vida de deshonor e impureza, como si, en lugar de la muerte inminente, se escoge la salvación de la vida a costa de cualquier tipo de prescripción previa. La muerte por los demás, si es libremente elegida y con ella se glorifican las propias creencias, es uno de los pilares de este tipo de personas. Sobre todo si esta muerte reparadora se produce para que su efecto redunde en favor de absolutos desconocidos. Por ende, Kaworu, muriendo en pos de un objetivo noble $-\mathrm{y}$ no solamente por el hecho de no importarle el acaecimiento o no del Tercer Impacto-, puede entonces adscribirse a esta caracterización de manera plena.

52 Ep. 24.19:34-20:08.

53 Cfr. Van Henten, J. W. The Maccabean martyrs as Saviours of the Jerwish People. A study of 2 and 4 Maccabees. JSJSup 57. Leiden, Brill, 1997, p. 136.

544 Ma.6.29, en Rahlfs, A. (ed.). Op. cit. 
Además de la centralidad que lleva inscrita en sí misma la muerte por causa de ideales elevados, se impone reseñar que los martirizados, por lo general, poseen otra característica por demás señera: su juventud. En efecto, estas personas no suelen ser de edad avanzada. La literatura ajena al contexto hebreo, puntualmente, brinda numerosos casos que pueden leerse en clave de favorecer este punto, tanto la tragedia griega como la lírica del mismo origen. De esta manera, los euripideos Ifigenia, ${ }^{55} \mathrm{Meneceo}^{56}$ y Políxena ${ }^{57}$ y los guerreros de las elegías de Calino ${ }^{58}$ y Tirteo $^{59}$ compartirán como principal particularidad una lozanía manifiesta, cosa que no hace más que magnificar el alcance de su gesto, pues entregan a la muerte muchos años por vivir. Esta clase de sacrificio, representado a los ojos divinos como un holocausto, ${ }^{60}$ ayuda a que se alcance el objetivo deseado, ya que las normas referentes a los sacrificios expiatorios prescribían ofrecer un buey joven ( $\mu$ ooxós ßoũ ) tanto por los pecados de los sacerdotes que representan al pueblo ${ }^{61}$ como por el pueblo mismo. ${ }^{62}$ Ahora bien, Eleazar efectivamente entrega su vida en circunstancias notables pero, en disonancia con el presente punto, es un anciano. ${ }^{63}$ De hecho, es esta ancianidad la que le permite presentarse a sí mismo como un ejemplo para las jóvenes generaciones y enfrentarse con autoridad a su torturador, evidentemente mucho más joven que él. Pero, en este caso, resulta obligatorio destacar que la lozanía de este sacerdote no se halla en su cuerpo, sino en su alma. Según lo que él mismo declara de sí, es su razón la que lo hace rejuvenecer ${ }^{64}$ puesto que, gracias a ella, su alma no ha sido mancillada por ninguna mala conducta. ${ }^{65}$ Además, que los demás torturados manifiesten que el elemento imperecedero de cada persona continúa su existencia más allá de la muerte ${ }^{66}$ aprueba la lectura posterior que establece su permanencia perenne junto a la divinidad ${ }^{67}$ en el mismo estado en el que dejan su recipiente corporal. Kaworu, así, pasará por una experiencia idéntica a la del anciano y sus sucesores. Adolescente en el momento de la narración - ya que de otro

55 E. $I A$ 433, en Diggle, J. (ed.). Euripidis Fabulae. Oxford, OUP, 1994.

56 E. Ph. 914, en idem.

57 E. Hec. 202, en idem.

58 1.18-19 W, en West, M. L. (ed.). Iambi et elegi Graeci Vol. 2. Oxford, OUP, 1972.

59 10.1-2 W, en idem.

60 Van Henten, Op. cit., p. 143.

${ }^{61}$ Lev. 4.3, en Rahlfs, A. (ed.). Op. cit.

62 Lev. 4.13, en idem.

634 Ma. 5.4, en idem.

64 4Ma. 5.31, en idem.

65 4Ma. 5.35, en idem.

66 4Ma. 9.8, en idem.

${ }^{67}$ SAyar, R. J. «"El temor del Señor es fuente de vida para escapar a los lazos de la muerte” (Pro. 12.19). La dicotomía alma/cuerpo en IV Macabeos", en Andrea, R. y G. Cadirola (comps.). Actas de las VII Jornadas Nacionales de Filosofía Antigua. El cuerpo y el alma en la filosofía antigua. Rosario, UNR Editora, 2015, pp. 261-267. 
modo no podría desempeñarse como piloto del Evangelion-, permanecerá justificadamente en ese estado ya que su vida — en tanto espíritu - es eterna y, al abandonar su cuerpo mortal, podrá conservar su estado juvenil cuando le sea el momento de regresar al mundo celestial.

A pesar de todo esto, aún es necesario un rasgo más para poder equiparar de manera efectiva al joven ángel con el estereotipo de mártir provisto por el anciano sacerdote hebreo, y es lo que el tratado que alberga a este último denomina fortaleza. Con semejante término se hace referencia tanto a la virilidad - y, por ende, a la valentía - como a la rectitud moral de la vida del individuo sometido a análisis. Ambas definiciones serán necesarias paralelamente en razón a su completa caracterización como ejemplo sacrificial. La primera será fácilmente verificable en el preciso momento de enfrentar las torturas y la posterior muerte, ya que es justamente gracias a ella que se entregan a tales padecimientos. La otra, de manifestación más compleja, saldrá a la luz en caso de que al condenado se le permita hablar y defender los motivos por los cuales prefiere el deceso antes que la vida y, con ellos, la moralidad de los pensamientos que sustentan esa opinión. Como en los tópicos anteriores, será en los casos de martirio narrados por el Libro IV de los Macabeos donde la valentía obtendrá estatus de virtud nodal puesto que todos los protagonistas de esa historia son calificados de tal manera y siempre reciben dicho apelativo una vez que ya han muerto. ${ }^{68}$ La causa de esa adjetivación, según este texto, obedece a que «su valentía se basó en aceptar lo bueno teniendo la posibilidad de escoger lo contrario». ${ }^{69}$ Este valor, entonces, hace que los hombres obren rectamente, ya que actuando así siguen los postulados de la más recta filosofía. ${ }^{70}$ Por lo tanto, su fundamento dialogará con la confianza que se tenga en la persistencia del alma en el otro mundo, receptáculo del reconocimiento divino.

En este caso, Kaworu pertenecería al grupo de los mártires debido a que ofrece su vida por el bien de la humanidad y además porque, de seguir vivo, las dos opciones que se le presentan como consecuentes a su acto de supervivencia son absolutamente negativas: o bien desencadenará el Tercer Impacto, ${ }^{71}$ o bien morirá a manos de «los viejos» por rebelarse contra ellos. ${ }^{72}$ Ambos, aunque no lo parezcan, son caminos intrínsecamente egoístas y mezquinos puesto que cualquiera que escoja seguir, técnicamente, lo llevará a una muerte en vano según los códigos morales que se desglosan

68 4Ma. 1.23; 5.23; 7.23; 15.10; 15.30, en Rahlfs, A. (ed.). Op. cit.

69 SAYAR, R. J. «"La palabra verdadera se instala para siempre” (Pro. 12.19). Identificaciones de léxico estoico en IV Macabeos", en Braicovich, R. et al. (comps.). Actas del Primer Simposio Internacional de Filosofía Helenística. Rosario 2013. En homenaje al Dr. Armando Poratti. Rosario, UNR Editora, 2013, p. 130.

70 Cfr. 4Ma. 1.16, en Rahlfs, A. (ed.). Op. cit.

71 73.20, en Sadamoto, Y. y Gainax. Op. cit.

7273.24 , en idem. 
del comportamiento del mártir modelo. De esta manera, de seguir el primero de los senderos posteriores y culminar así con el pacto establecido con SEELE, la humanidad sufriría un mal por su causa, puesto que dejaría de existir como tal. La segunda salida no es mucho mejor en tanto se haría un mal a sí mismo, cuyas consecuencias serían nefastas. Es decir, en este caso, su propio deceso traería aparejado el Tercer Impacto, que sucedería de todos modos, impulsado por el ambicioso Gendo Ikari. ${ }^{73}$ La valentía del joven, entonces, estriba en abrazar una causa plenamente humana y actuar en favor de ella dado que esto sería lo «correcto» según los cánones de justicia que comparten la esfera celestial y la mundana.

No obstante, para juzgar qué es lo conveniente o lo justo en esta delicada situación en la que él y los que lo rodean se hallan no resulta desacertado recordar que lo que debería hacer Kaworu no es estrictamente parte del plan divino sino de una maquinación humana fraguada para quitar de su sitial a la divinidad. ${ }^{74}$ Así, su sacrificio a favor de la Creación y de los seres que la forman toma el cariz de mandato divino: realizándolo no solo haría fracasar la soberbia humana sino que, además, se convertiría en un ejemplo para las personas directamente implicadas en su propia muerte. De este modo, el mandato que les indica a las personas virtuosas que «transgredir la Ley es igual de grave en asuntos pequeños o grandes» ${ }^{75}$ instituye como consecuencia que la Ley es capaz de enseñar todas las virtudes, de modo de adorar al Señor de la manera que le es agradable. ${ }^{76}$ Hacer lo correcto, entonces, es una demostración de sabiduría y de racionalidad, dado que negar los mandatos de Dios es negar la propia sensatez. ${ }^{77}$ Kaworu, entonces, no haría más que tomar en sus manos la esperanza de la humanidad, ${ }^{78}$ pero no para hacer la voluntad de esta última, sino la de Dios, entregándose como víctima para que esté al alcance de los perpetradores del Plan de Consumación comprender las consecuencias de lo que pretenden realizar. La única esperanza restante ${ }^{79}$ residiría, entonces, no en renacer como una nueva forma de vida, sino en reconocer su propia soberbia y actuar en consecuencia para evitar el desenlace fatal; cosa que no deja de ser, en todos los aspectos, sumamente trágica. Es por todo lo precedente que Kaworu se plantearía a sí mismo, conscientemente, como ejemplo - del mismo modo en que lo hará Eleazar - ${ }^{80}$ pero ya no para toda la humanidad, sino únicamente para Shinji. ${ }^{81} \mathrm{El}$ hijo del Comandante, más adelante en la historia,

\footnotetext{
$7375.10-11$ y 76 y ss. ,en idem.

74 78.13, en idem.

75 4Ma. 5.20, en Rahlfs, A. (ed.). Op. cit.

76 Cfr. 4Ma. 5.24, en idem.

77 SAYar, R. J. Op. cit., p. 133.

78 71.27, en Sadamoto, Y. y Gainax. Op. cit.

79 71.26, en idem.

80 Cfr. 4Ma. 6.19, en Rahlfs, A. (ed.). Op. cit.

81 74.12, en Sadamoto, Y. y Gainax. Op. cit.
} 
es quien se convertirá en el juez del destino futuro de la raza humana. Kaworu, que lo intuye, se entrega para permitirle al joven Ikari «encontrar su lugar en el mundo». ${ }^{82}$ El saber que su forma humana solo obedece a la necesidad que se tiene de él para alcanzar un fin «sacrílego» solo ahonda la equiparación que es posible realizar con la sabiduría de los que lo precedieron, pues la voluntad divina pasa a primer plano y la ejemplificación que de eso se sigue toma un puesto de importancia suma. Es decir que, de no ser por este sacrificio, Shinji no se vería movido a la reflexión y, en consecuencia, no podría ver las bondades de este caótico mundo (94.8) y no se sentiría motivado a restablecerlo. El orbe renacido, en consecuencia, tendría siempre en cuenta el sacrificio de Kaworu y sus causas y ayudaría a sus habitantes a verlo siempre de manera positiva. ${ }^{83}$

\section{A modo de reflexiones finales}

Con el punteo precedente y los elementos compartidos entre las tradiciones del medio oriente antiguo retomadas por Israel y extendidas por el mundo antiguo a través de la Diáspora y la difusión de la lengua griega, será posible entonces demostrar de manera sistemática tanto la naturaleza angélica como la condición de mártir que ostentaría Kaworu, pues cumpliría satisfactoriamente todas las características que los respectivos paradigmas establecen como esperables para cada uno de ellos. De este modo, las marcas reseñadas conformarán un compendio de las características que hacen que un mártir efectivamente lo sea y, en este caso particular, que se conjunten con los rasgos angélicos trasvasados a lo largo de la tradición religiosa asirio-persa. Es cierto, no obstante, que es imposible conjeturar un paradigma partiendo de un único ejemplo. El schema martyrorum propuesto se justifica en tanto Eleazar ejemplifica de un modo particularmente explícito y concreto las aristas que componen semejante modelo. Por este motivo, además, es que, de entre las dos versiones de la historia de su sacrificio, se optó por ahondar la comparación con la que se presenta en $I V$ Macabeos debido a que es más extensa - y, por ende, más detallada - que la narrada en II Macabeos. Únicamente gracias a esto - y a la temática propia del libro- será posible descubrir los pormenores filosóficos de esta conducta. La equivalencia de Kaworu con un mártir judeocristiano favorecido por su verdadera condición será, de esta manera, prácticamente transparente. Es cierto que el resto de los elementos en la serie pueden carecer completamente de una correlación o función específica asociada a estos credos y tratarse efectiva y únicamente de elementos de verosimilitud, cuya presencia solo apuntaría a construir la historia vinculándola a una cuestión determinada que poco tenga que ver

${ }^{82}$ Gajardo Gonzalez, R. A. El Cristianismo en la animación japonesa [Tesis de Licenciatura]. Universidad Academia de Humanismo Cristiano, Escuela de Periodismo, Santiago de Chile, 2011, p. 183.

83 96, passim, en Sadamoto, Y.y Gainax. Op. cit. 
con la tradición religiosa. ${ }^{84}$ Pero, como dijo acertadamente el director de este «mito de los noventa»,

Evangelion es como un rompecabezas. Cualquier persona puede verlo y dar su propia respuesta. Es decir que estamos ofreciéndole a los espectadores la oportunidad de pensar por ellos mismos, así cada uno puede imaginarse su propio mundo. Nunca vamos a ofrecer ningún tipo de respuesta [...]. No esperen recibir respuestas de nadie. No esperen ser guiados todo el tiempo. Todos tenemos que encontrar nuestras propias respuestas. ${ }^{85}$

Por lo tanto, será posible afirmar — con estas lecturas más que avaladas- que, en la línea de esta lectura, Nagisa Kaworu no es solo un mártir en el sentido actual del término sino que su cualidad de tal se verifica dentro de la serie, tanto por las evidencias que ocuparon el meollo de este estudio como por las consecuencias que conlleva. Ya que es puntualmente este sacrificio el que abre la puerta a las preguntas que Shinji se hará a lo largo de los últimos capítulos - mientras se desarrolla el Tercer Impacto- acerca de las dificultades de la existencia y de la capacidad que puede tener cada persona - y, sobre todo, Ikari- de aceptarse a sí mismo y del modo en que se desenvuelve en su entorno. Así las cosas, el mito del sacrificio autoinfringido, como tal, se volverá un modo de significación particular, ya que no lo será de la variedad de conceptos que lo componen sino de lo que todos ellos finalmente expresan en conjunto en el plano del signo. Es decir que no se abordará solamente como un elemento que se produce por circunstancias de creación y de recepción análogas, ${ }^{86}$ que en este caso tendrán su correlato en un grupo de sujetos, ignotos entre sí, que a lo largo de las eras han compartido una serie de conductas que implican la necesidad de la muerte ante la amenaza de cierto grupo de valores. En cambio, se observará una pretensión de ejemplaridad en aras de un objetivo superior transmitida linealmente entre quienes la ejecutan. El deceso en defensa de los propios ideales, en tanto signo, se vuelve significante de un sistema mayor en el cual su significado posee una doble lectura. La principal de estas interpretaciones expondrá que tal acción es una conducta digna de ser imitada por todos los que compartan los valores de quien se sacrifica. La lectura subordinada entenderá que a este sacrificio le sigue indefectiblemente una mirada de benevolencia de la divinidad a quien previamente - a título personal o por la comunidad en su conjunto- se ha ofendido. Empero, tal como se ha demostrado a lo largo de estas líneas, Nagisa no se ofrece para reconciliar a Dios con los humanos sino para que Shinji sea plenamente consciente de la necesidad de que el mundo siga su curso cuando se llegue a la cima de la rebeldía humana. De modo similar con lo que ocurre con algunos de los consumidores de este tipo de productos culturales, es preciso que ocurra esta suerte de anagnórisis para poder lograr un profundo cambio a

${ }^{84}$ Gajardo González, R. A. Op. cit., p. 102.

85 Sadamoto, Y. y Gainax. Op. cit., p. 6.

${ }^{86}$ Cfr. Guillén, C. The Challenge of Comparative Literature. Cambridge-London, Harvard University Press, 1993, p. 192. 
nivel personal en lo que se refiere a la inserción social del sujeto. El mito salvífico, por lo tanto, se propone a sí mismo como una salida posible a tal atolladero. Si el sacrificio es posible, aun a costa de dejar de lado la propia «zona de confort» —es decir, en el caso de Tabris, su naturaleza angélica-, ha de practicarse en pos de una comprensión más cabal del mundo y de la mejor manera de introducirse en él.

Finalmente, la construcción martirial que se levanta en torno a Nagisa, gracias a los vínculos que pueden trazarse teniéndola como punto de llegada y de partida de sucesivas intelecciones, será una herramienta de lo más adecuada para acabar de vincular a la serie con sus pretendidas fuentes gnósticas. Así como esta corriente ofrece la explicación más puntual al evento que ha de suceder al final de los tiempos, cuando indefectiblemente todos los vivientes volverán a ser uno; del mismo modo el sacrificio de uno de los individuos antes de este momento figurará la salvación del todo social en su conjunto. Por ende, la vinculación entre Dios y el hombre, centro de toda la obra, se hará más cercana y la invasión de los ángeles se comprenderá de otro modo, dado que estos últimos estarán más dispuestos a ofrecer su vida antes que fracasar en su misión. La visión del mundo judeocristiana tomará forma en estos últimos y los hará no solo más humanos que los propios hombres sino incluso más misteriosos, dada la carga de incógnitas ontológicas que poseen todos ellos. La pregunta de Shinji acerca de la exigencia de enfrentarlos, repasando los elementos de la presente argumentación, se responderá sola habida cuenta del devenir de los acontecimientos. En términos simbólicos, NERV se posicionará lenta e inexorablemente del lado del «hombre» en tanto criatura tendiente al «cálculo» y a la consecuente «rebeldía», mientras que los ángeles y luego Rei y Shinji lo harán del lado de «Dios», dispuestos a salvar a la humanidad de sí misma y a reeducarla para seguir viviendo en comunidad.

La figura del mártir y sus vínculos con la literatura sacra de cuño hebreo serán, en consecuencia, una figura siempre fértil para marcar esta necesidad de cambio. $Y$ es por eso que la profundización del vínculo con una tradición religiosa «extraña» resulta necesaria. Solo de esa manera se produciría la concientización de estos problemas y su consecuente abordaje. Nagisa debe ser un mártir tanto para aplicar las lógicas del mito en el ámbito intradiegético como para expandirlas hacia sus consumidores. Es cierto que no todas las interpretaciones del signo mitológico serán aplicables en situaciones «reales». Pero una de ellas, de particular singularidad, será sumamente apta para caracterizar los dramas humanos y sociales del Tokyo finisecular. Así como el ángel no tuvo empacho en dejar de lado su naturaleza angélica para mostrar una conducta ejemplar para con los hombres, de manera que estos logren estar dispuestos a cambiar la visión de cuanto los rodea para, con ojos nuevos, ser parte de ese todo mayor — simbolizado por Shinji- que intenta llevar el mundo adelante. La comprensión de este último punto clarificará las escenas finales hasta el punto en que el piloto del Evangelion.01 se identifique como tal y conserve, con todas sus falencias pero iluminado de otro modo, es(t)e mundo en el que vive. 


\section{Bibliografía}

BAne, T. Encyclopedia of Demons in World religions and cultures. Jefferson, McFarland, 2012.

Boll, F. (ed.). Codices germanici [Catalogus codicum astrologum Graecorum 7]. Brussels, Lamertin.

Breen, J. «Shinto and Christianity: A History of Conflict and Compromise», en Mullins, M. R. (ed.). Handbook of Christianity in Japan, Leiden-Boston, Brill, 2003, pp. 249-276.

Breen, J. y Teeuwen, M. A new bistory of Shinto. Chichester, Wiley-Blackwell, 2010.

Совоs, T. «Animación japonesa y globalización: la latinización y la subcultura otaku en América Latina», en Razón y palabra, n. 72 (2010). Disponible en: http://www.razonypalabra.org.mx/N/N72/Varia 72/32 Cobos 72.pdf

Davidson, G. A Dictionary of Angels, including the fallen angels. New York, The Free Press, 1966.

De Santos Otero, A. (ed.). Los Evangelios Apócrifos. Madrid, BAC, 2005.

Diggle, J. (ed.). Euripidis Fabulae. Oxford, OUP, 1994.

Eco, U. Apocalipticos e integrados. Buenos Aires, Sudamericana, 2013 [1964].

Ferretti, V. «Emperor Reigen and the Change in Court-Bakufu Relations since the Tenna to the Kyōtō Era», en East and West, n. ${ }^{\circ}$ 1/2, (1996), pp. 167-174.

Gajardo González, R. A. El Cristianismo en la animación japonesa [Tesis de Licenciatura]. Universidad Academia de Humanismo Cristiano, Escuela de Periodismo, Santiago de Chile, 2011.

Giovino, M. The Assyrian Sacred Tree: A history of Interpretations. Fribourg/Göttingen, Academic Press Fribourg/Vandenhoeck \& Ruprecht, 2007.

Guevara de Álvarez, M. E. (ed.). Antología gnómica de la literatura griega. Liricos arcaicos (poetas elegiacos y yambógrafos). Buenos Aires, Santiago Arcos, 2014.

Guillén, C. The Challenge of Comparative Literature. Cambridge-London, Harvard University Press, 1993. 
Heine, S. «From Rice Cultivation to Mind Contemplation: The Meaning of Impermanence in Japanese Religion», en History of Religions, n. ${ }^{\circ} 30 / 4$ (1991), pp. 373-403.

Van Henten, J. W. The Maccabean martyrs as Saviours of the Jerwish People. A study of 2 and 4 Maccabees. JSJSup 57. Leiden, Brill, 1997.

Ingulsrud, J. E. y Allen, K. Reading Japan Cool. Patterns of Manga Literacy and Discourse. New York, Lexington Books, 2009.

Kelly, S. y Rogers, R. Who in hell. A guide to the whole damned bunch. Michigan, Villard, 1996.

Leibovici, M. «Génies et démons en Babylonie», en VV.AA. Génies, anges et démons, Paris, Seuil, 1971.

Levi, J. Los funcionarios divinos. Politica, despotismo y mistica en la China antigua. Madrid, Alianza, 1991.

LÉvy, É. Transcendental magic. York Beach, Weiser, 2001.

Liverani, M. El antiguo oriente. Barcelona, Crítica, 1995.

López Salvá, M. «Demonios y espíritus en las religiones orientrales primitivas», en Pérez Jiménez, A. y Cruz Andreotti, G. (eds.). Seres intermedios. Ángeles, Demonios y Genios en el Mundo Mediterráneo. Madrid, Ediciones Clásicas, 2000.

Mann, M. Las fuentes del poder social, I. Madrid, Alianza, 1991.

Miyazaki, K. «Roman Catholic Mission in Pre-Modern Japan», en Mullins, M. R. (ed.). Handbook of Christianity in Japan. Leiden-Boston, Brill, 2003, pp. 1-18.

Naumann, N. Antiguos mitos japoneses. Barcelona, Herder, 1999.

Отто, R. Lo santo. Lo racional y lo irracional en la idea de Dios. Madrid, Alianza, 1998.

Pérez Jiménez, A. y Cruz Andreotti, G. (eds.). Seres intermedios. Ángeles, Demonios y Genios en el Mundo Mediterráneo. Madrid, Ediciones Clásicas, 2000.

Rahlfs, A. (ed.). Septuaginta, id est Vetus Testamentum graecae iuxta LXX interpretes Vol. 1-2. Stuttgart, Württembergische Bibelanstalt, 1971. 
Rubio, C. y Moratalla, R. T. «Introducción», en Oo no Yasumaro (comp.). Kojiki. Madrid, Trotta, 2008.

貞本義行 - GAINAX 新世紀エヴアンゲリオン. 東京, 角川書店, 2004.

Sadamoto, Y. y Gainax. Evangelion. Buenos Aires, Ivrea, 2004. Traducción de Agustín Gómez Sanz.

SAYAR, R. J. «"El temor del Señor es fuente de vida para escapar a los lazos de la muerte" (Pro. 12.19). La dicotomía alma / cuerpo en IV Macabeos», en AndreA, R. y G. Cadirola (Comps.). Actas de las VII Jornadas Nacionales de Filosofia Antigua. El cuerpo y el alma en la filosofía antigua. Rosario, UNR Editora, 2015, pp. 261-267.

— “La palabra verdadera se instala para siempre” (Pro. 12.19). Identificaciones de léxico estoico en IV Macabeos», en Braicovich, R. et al. (comps.). Actas del Primer Simposio Internacional de Filosofía Helenistica. Rosario 2013. En homenaje al Dr. Armando Poratti. Rosario, UNR Editora, 2013, pp. 128-135.

Teeuwen, M. y Schied, B. «Tracing Shinto in the History of Kami Worship», en Japanese Journal of Religious Studies, vol. 29/3-4, (2002), pp. 195-207.

Trigger, B. Understanding Early Civilizations. A Comparative Study. Cambridge, Cambridge University Press, 2003.

VV.AA. Apócrifos del Antiguo Testamento IV: Ciclo de Henoc. Madrid, Cristiandad, 1984.

Vitaliano, D. Legends of the Earth. Bloomington, Indiana University Press, 1973.

WEst, M. L. (ed.). Iambi et elegi Graeci, vol. 2. Oxford, OUP, 1972. 